\title{
Quelles évolutions pour la consommation d'énergie domestique et la gestion des ressources naturelles dans les bassins d'approvisionnement des grandes villes du Sud?
}

\author{
Régis Peltier ${ }^{1,2}$ \\ ${ }^{1}$ Cirad \\ Forêts et Sociétés \\ Campus international de Baillarguet \\ 34398 Montpellier \\ France \\ 2 Forêts et Sociétés \\ Univ Montpellier \\ Cirad \\ Campus international de Baillarguet \\ Montpellier \\ France

\section{Auteur correspondant /} \\ Corresponding author: \\ Régis PELTIER - \\ regis.peltier@cirad.fr
}

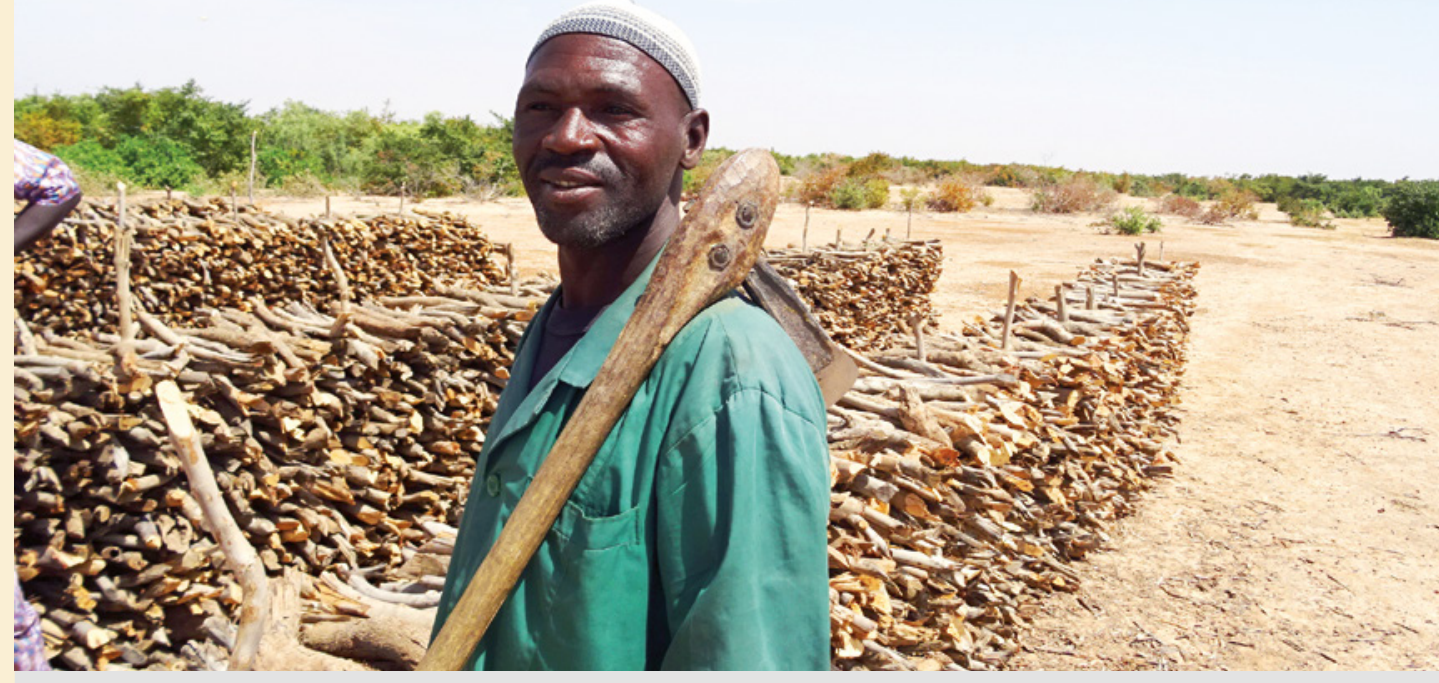

Photo 1.

Bûcheron d'un marché rural de bois-énergie au Sud-Niger. Fondé en 1992 par le projet BM Énergie II, ce marché rural fonctionne toujours en 2019 avec l'appui du projet FFEM-AFD Fonabes et il respecte toujours son quota annuel de stères de bois exploités et vendus. La productivité du massif se maintient mais sa biodiversité a diminué.

Photo R. Peltier.

Doi : 10.19182/bft2019.340. a31708 - Droit d'auteur @ 2019, Bois et Forêts des Tropiques @ Cirad - Date de soumission : 29 septembre 2018 ; date d'acceptation : 8 janvier 2019 ; date de publication : $1^{\mathrm{er}}$ avril 2019.

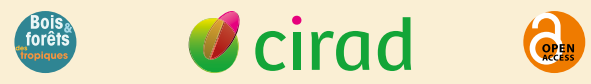

(c) $(1) \Theta$
Citer l'article / To cite the article

Peltier R., 2019. Quelles évolutions pour la consommation d'énergie domestique et la gestion des ressources naturelles dans les bassins d'approvisionnement des grandes villes du Sud ? Bois et Forêts des Tropiques, 340 : 3-12. Doi : https://doi.org/10.19182/bft2019.340.a31708 


\section{RÉSUMÉ}

\section{Quelles évolutions pour la consommation d'énergie domestique et la gestion des ressources naturelles dans les bassins d'approvisionnement des grandes villes du Sud?}

À l'occasion de la parution de ce numéro de Bois et Forêts des Tropiques, son coordonnateur tire un bilan des recherches-actions que lui-même et les équipes partenaires ont consacrées au bois-énergie en Afrique, pendant une quarantaine d'années. Il propose un schéma prospectif, pour expliquer en quoi ont été complémentaires ces actions, certaines portant sur la foresterie, l'agroforesterie, la bioénergie, et d'autres sur le social, l'économie, le droit, la fiscalité ou les politiques forestières. Il montre que, du fait de la démographie, les besoins en bois-énergie ont rejoint, puis dépassé, la productivité des bassins d'approvisionnement des grandes villes sahéliennes, au cours des années 1990. Par la suite, les services gouvernementaux et les ONG ont mené diverses tentatives et projets pour diminuer la consommation de bois-énergie, mieux gérer les formations naturelles et restaurer les systèmes agroforestiers. Ceci a permis de freiner la dégradation des ressources naturelles, de satisfaire les besoins des populations urbaines en énergie domestique et de fournir des revenus aux populations rurales. Cependant, cette dégradation est presque partout toujours en cours et la consommation est satisfaite au prix de la dégradation du capital productif des forêts. Pour l'avenir, l'hypothèse a été émise que, pour éviter des troubles sociaux urbains liés à la pénurie d'énergie domestique, tout en continuant à garantir des revenus aux ruraux, il est indispensable de combiner des politiques d'économie d'énergie, de restauration et de gestion des écosystèmes et des systèmes agroforestiers. II faut substituer la partie de l'énergie domestique qui dépasse les capacités de production des bassins d'approvisionnement en bois-énergie. Ceci nécessitera de faire évoluer les politiques nationales sur le foncier, les taxes et les subventions, et d'établir des schémas régionaux d'aménagement.

Mots-clés : bois-énergie, gestion durable des formations naturelles, agroforesterie, économie d'énergie domestique, substitution d'énergie, politique forestière, foncier, schémas régionaux d'aménagement, tropiques, zone sèche et aride.

\section{ABSTRACT}

\section{What are the evolutions for domestic energy consumption and natural resource management in the supply basins of the large cities of the South?}

For this issue of Bois et Forêts des Tropiques, the editor reviews the research and activities on fuelwood that he and his team have undertaken in Africa over the last forty years. A forward scenario is proposed that explains how these activities - some focusing on forestry, agroforestry and biofuels, others on social, economic and legal aspects - have complemented each other. He shows that in the 1990s, for demographic reasons, demand for fuelwood first caught up with and then exceeded the productive capacity of regions supplying large cities in the Sahel. Attempts were subsequently made by various government departments, NGOs and other projects to reduce fuelwood consumption, improve the management of natural forest stands and restore agroforestry systems. Although these attempts have slowed down the degradation of natural resources and are satisfying urban needs for domestic fuel while providing income for rural families, degradation is continuing almost everywhere and demand is only being met by depleting productive forest capital. The proposal made for the future is that in order to avoid social unrest in cities due to domestic fuel scarcities while continuing to guarantee an income for rural people, it is essential to combine energy efficiency policies with measures to restore and manage agroforestry systems. The share of domestic fuel that exceeds the productive capacities of fuelwood supply basins has to be substituted, and this will demand changes in national policies on land, taxation and subsidies and the introduction of regional planning measures.

Keywords: fuelwood, sustainable management of natural forests, agroforestry, domestic fuel savings, energy substitution, forest policy, land, regional planning, tropics, arid and dry zones.

\section{¿Alguna evolución para el consumo de energía doméstica y la gestión de los recursos naturales en las cuencas de aprovisionamiento de las grandes ciudades del Sur?}

Con motivo de la aparición de este número de Bois et Forêts des Tropiques, su coordinador hace balance de las investigaciones-acciones que él mismo y los equipos asociados han dedicado a la leña en África durante una cuarentena de años. Ofrece un esquema prospectivo para explicar en qué han sido complementarias estas acciones, algunas de las cuales tratan sobre silvicultura, agrosilvicultura o bioenergía, y otras sobre sociología, economía, derecho, fiscalidad o políticas forestales. Muestra que, por razones demográficas, las necesidades de leña alcanzaron, y después superaron, la productividad de las cuencas de aprovisionamiento de las grandes ciudades del Sahel en el curso de los años 1990. A continuación, servicios gubernamentales y ONG llevaron a cabo varias tentativas y proyectos para disminuir el consumo de leña, gestionar mejor las formaciones naturales y restaurar los sistemas agroforestales. Ello ha permitido frenar la degradación de los recursos naturales, satisfacer las necesidades de energía doméstica de estas poblaciones urbanas y proporcionar recursos para las poblaciones rurales. Sin embargo, esta degradación se está produciendo casi por todas partes, y el consumo se satisface pagando el precio del deterioro del capital productivo de los bosques. Para el porvenir, se ha establecido la hipótesis de que, para evitar alteraciones sociales urbanas relacionadas con la escasez de energía doméstica y continuar garantizando los ingresos de las poblaciones rurales, es indispensable combinar políticas de economía de energía, de restauración y de gestión de ecosistemas y sistemas agroforestales. Hay que sustituir la parte de la energía doméstica que supera las capacidades de producción de las cuencas de aprovisionamiento de leña. Ello requerirá hacer evolucionar las políticas nacionales sobre la propiedad, las tasas y las subvenciones, y establecer esquemas regionales de ordenación.

Palabras clave: leña, gestión sostenible de las formaciones naturales, agrosilvicultura, economía de energía doméstica, sustitución energética, política forestal, propiedad, esquemas regionales de ordenación, trópicos, zona seca y árida. 


\section{Approvisionner en bois-énergie : une recherche-action de longue haleine}

Bientôt au terme d'une carrière d'une quarantaine d'années, en grande partie consacrée à des recherches-actions autour du bois-énergie en Afrique ${ }^{1}$, j'ai souhaité coordonner un numéro spécial de la revue Bois et Forêts des Tropiques sur ce même thème. Le but était d'en tirer un bilan, fût-il très partiel, et d'ouvrir des pistes d'avenir.

Pendant ma carrière, avec mes collègues chercheurs et développeurs, nous avons étudié, par enquêtes, les filières bois-énergie de nombreuses grandes villes du Sahel et d'Afrique centrale et nous en avons montré l'importance économique pour des centaines de milliers de travailleurs, en particulier en milieu rural (photos 1 et 2). Nous avons caractérisé, par inventaire et télédétection, l'état des formations végétales où ce bois était prélevé, et montré leur dégradation. Nous avons testé des solutions pour réduire la consommation de bois ou de charbon par diffusion de foyers améliorés ou appuis financiers à la substitution d'énergie. Nous avons mené des projets de gestion des territoires ruraux, en privilégiant le diagnostic partagé de l'état des ressources naturelles et la recherche de solutions communes et individuelles pour les restaurer lorsque cela s'avérait possible. Nous avons co-construit avec les villageois des modèles techniques de plantations, d'agroforesterie ou de régénération des écosystèmes naturels. Nous avons amélioré les techniques de carbonisation (photo 3). Nous avons été à l'origine ou sommes venus en appui à des modifications législatives ou fiscales et à la mise en place de mécanisme de subvention pour freiner la dégradation et favoriser la restauration. Enfin, l'ensemble de ces travaux a souvent été coordonné sous la forme d'un Schéma directeur d'approvisionnement des villes en énergie domestique (SDAED).

Cependant, il nous manquait un cadre conceptuel pour expliquer facilement en quoi pouvaient être complémentaires ces actions très différentes, certaines très techniques portant sur la foresterie, l'agroforesterie ou la bioénergétique, d'autres sur le social, l'économie, le droit, la fiscalité ou les politiques nationales ou internationales. Pour nos interlocuteurs des gouvernements, des ONG, de l'aide internationale,

R. Peltier a notamment travaillé sur les plantations communales ou privées au Burundi, les plantations étatiques ou privées, l'agroforesterie et le sylvo-pastoralisme au Nord-Cameroun, la foresterie communautaire au Niger et au Mali, l'approvisionnement de centrales à biomasse à Madagascar et l'approvisionnement en bois d'une mégapole en République démocratique du Congo. pour des journalistes, des étudiants ou de simples citoyens, nos actions semblaient souvent disparates, voire contradictoires. Nous étions harcelés de questions du type : « comment prétendez-vous protéger la forêt en organisant les bûcherons au sein d'organisations de foresterie communautaire : est-ce que vous n'allez pas encourager la déforestation ? "; " pourquoi êtes-vous contre l'interdiction d'utilisation du bois-énergie ? "; " est-ce que vous n'allez pas enfermer les bûcherons dans des pièges à pauvreté ?».

C'est pourquoi, au cours du colloque international intitulé « Gestion des ressources forestières des territoires périurbains et changement climatique ${ }^{2}$ », Aboubacar Ichaou et moimême avons dessiné un schéma prospectif (Ichaou et Peltier, 2017) que j'ai présenté et fait évoluer au cours de plusieurs réunions, dont la conférence « Wood: an ever-present domestic energy priority for people in emerging Africa ${ }^{3}$ (Marien et al., 2018). Bien que ce graphique ait été présenté et discuté oralement, il n'a pas été, jusqu'à ce jour, publié ni expliqué dans aucune revue.

J'ai donc profité de la publication de ce numéro spécial pour en publier la version actuelle dans le présent éditorial (figure 1), pour en expliquer les différents segments, pour faire émerger les hypothèses globales qu'il sous-tend, puis, enfin, pour analyser brièvement en quoi les articles de ce numéro confirment ou infirment le modèle et les hypothèses. Les dates, sur l'axe des abscisses (x), et les quantités d'énergie, sur celui des ordonnées (y), n'ont qu'une valeur indicative.

\footnotetext{
${ }^{2}$ Ce colloque a eu lieu à Niamey (Niger) du 31 octobre au 2 novembre 2017.
}

3 International Conference on Sustainable Energy for Africa, Bruxelles, 23-25 octobre 2017.

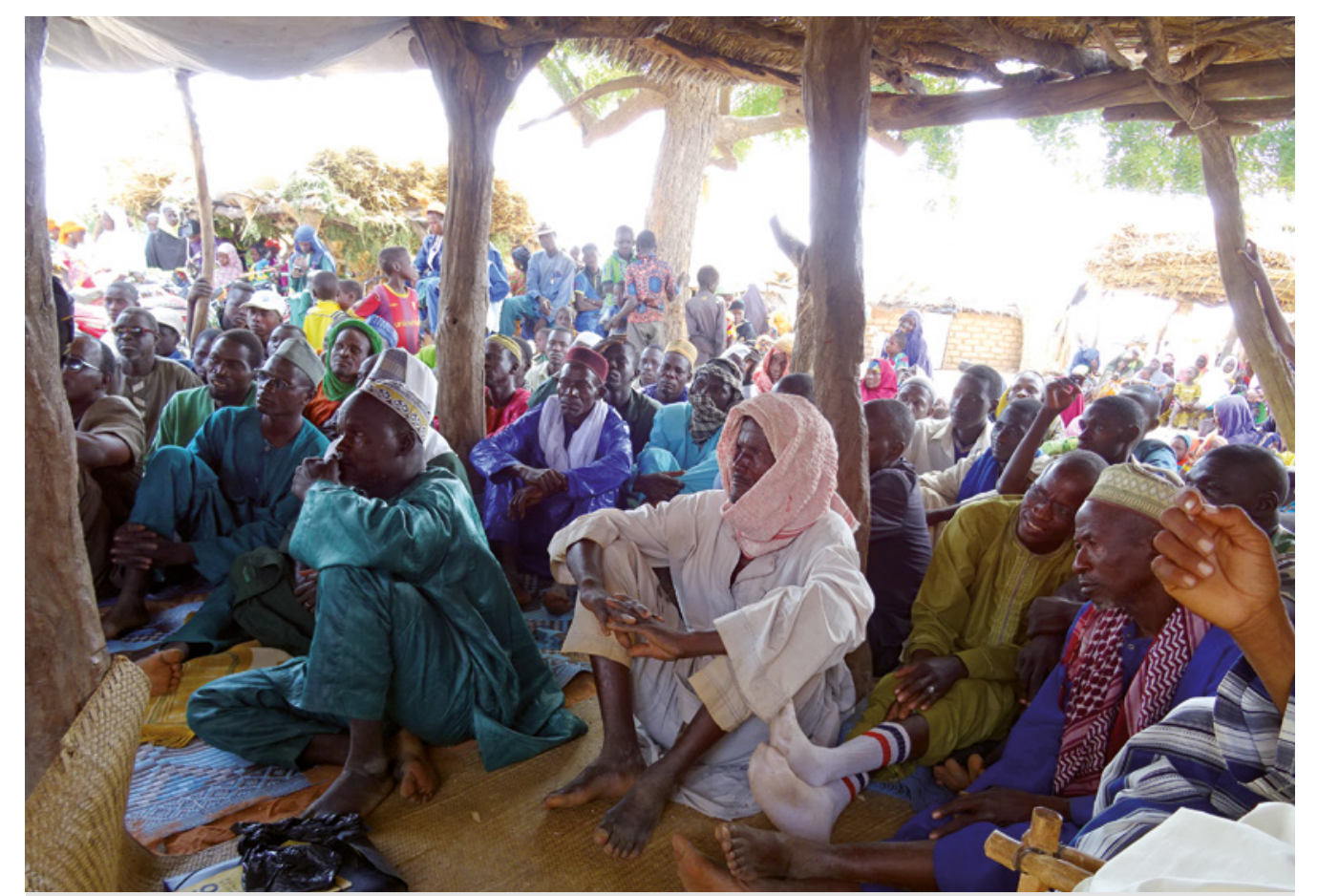

Photo 2.

Reunion du comité villageois en charge de la gestion d'un marché rural de bois-énergie au Sud-Niger (notables au premier plan de la photo). Les jeunes (au second plan) sont certainement ceux qui ont le plus profité de la manne financière apportée par le marché rural (vaccination infantile, maison de santé, eau potable, construction et fonctionnement d'une école), mais accepteront-ils de travailler dans des conditions aussi dures que leurs parents? Photo R. Peltier. 


\section{De 1955 à 1995 : les besoins en bois-énergie rejoignent puis dépassent la productivité des bassins d'approvisionnement des grandes villes sahéliennes}

Avec l'augmentation de la population des villes et des campagnes sahéliennes, appelée à doubler dans les 30 dernières années, les besoins en énergie domestique ont augmenté considérablement (Bachard, 2005). Dans les années 1970, la plupart des villes consommaient du bois récolté dans un bassin d'environ $100 \mathrm{~km}$ de rayon. La consommation de bois par habitant restait à peu près constante, de l'ordre de $1 \mathrm{~kg} / \mathrm{hab}$./jour, et la consommation par ville augmentait donc proportionnellement à celle de sa population (figure 1 , segment [AA']) (Madon et al., 1985). Cependant, par la suite, plus les villes ont grossi, plus la demande en bois-énergie a augmenté et plus les lieux de coupe se sont éloignés. Il est devenu plus rentable économiquement, pour les grossistes transporteurs, de transformer le bois en charbon (Gazull, 2009). Cette transformation a augmenté le niveau de consommation en tonnes équivalent bois (teb) par habitant de la ville (Gazull et Gautier, 2014). Par conséquent, la consommation en teb a augmenté plus rapidement que le nombre d'habitants (figure 1 , segment [A'A"] intégrant le passage au charbon de bois, et segment [A"B] intégrant l'éloignement des lieux de récolte, donc l'augmentation de l'énergie nécessaire au transport du charbon) (photo 4).
De 1955 à 1975, la grande majorité de cette énergie domestique était fournie par les espaces forestiers, sous forme principalement de bois et, très localement, de charbon de bois. Ce bois était prélevé dans un rayon d'environ $100 \mathrm{~km}$ autour des villes. À l'intérieur d'un cercle d'une vingtaine de kilomètres autour des villes, les zones boisées étaient facilement accessibles pour le transport non mécanisé. De ce fait, les formations arborées étaient très sollicitées par l'exploitation forestière, et donc très dégradées (Peltier et al., 1994). Mais au-delà, la productivité en bois-énergie de ces espaces forestiers très résilients était peu altérée et pouvait être considérée comme constante (figure 1, segment [EE']). Par exemple, pour ce qui concerne les formations du sud du Niger, Ichaou (2004) et Peltier et al. (2009) ont montré que leur productivité en bois-énergie se maintenait, même avec un régime de taillis à courtes révolutions (5-6 ans), malgré le fait que leur biodiversité se dégradait. De 1975 à 1995, la productivité des bassins d'approvisionnement a progressivement augmenté par agrandissement du rayon de prélèvement, jusqu'à environ $150 \mathrm{~km}$ autour des villes (figure 1, segment [E'B]).

C'est aux alentours de l'année 1995 que la consommation de bois-énergie de grandes villes comme Niamey a égalé la productivité de leur bassin d'approvisionnement (figure 1, point B).

La flèche verticale verte représente la différence entre la capacité de production en bois-énergie, ou « productivité " des ressources naturelles du bassin d'alimentation de la ville, et ses besoins en énergie domestique, exprimés dans la même unité, par exemple en tonnes équivalent bois (teb). La couleur verte a été choisie pour exprimer que cette différence est encore positive.

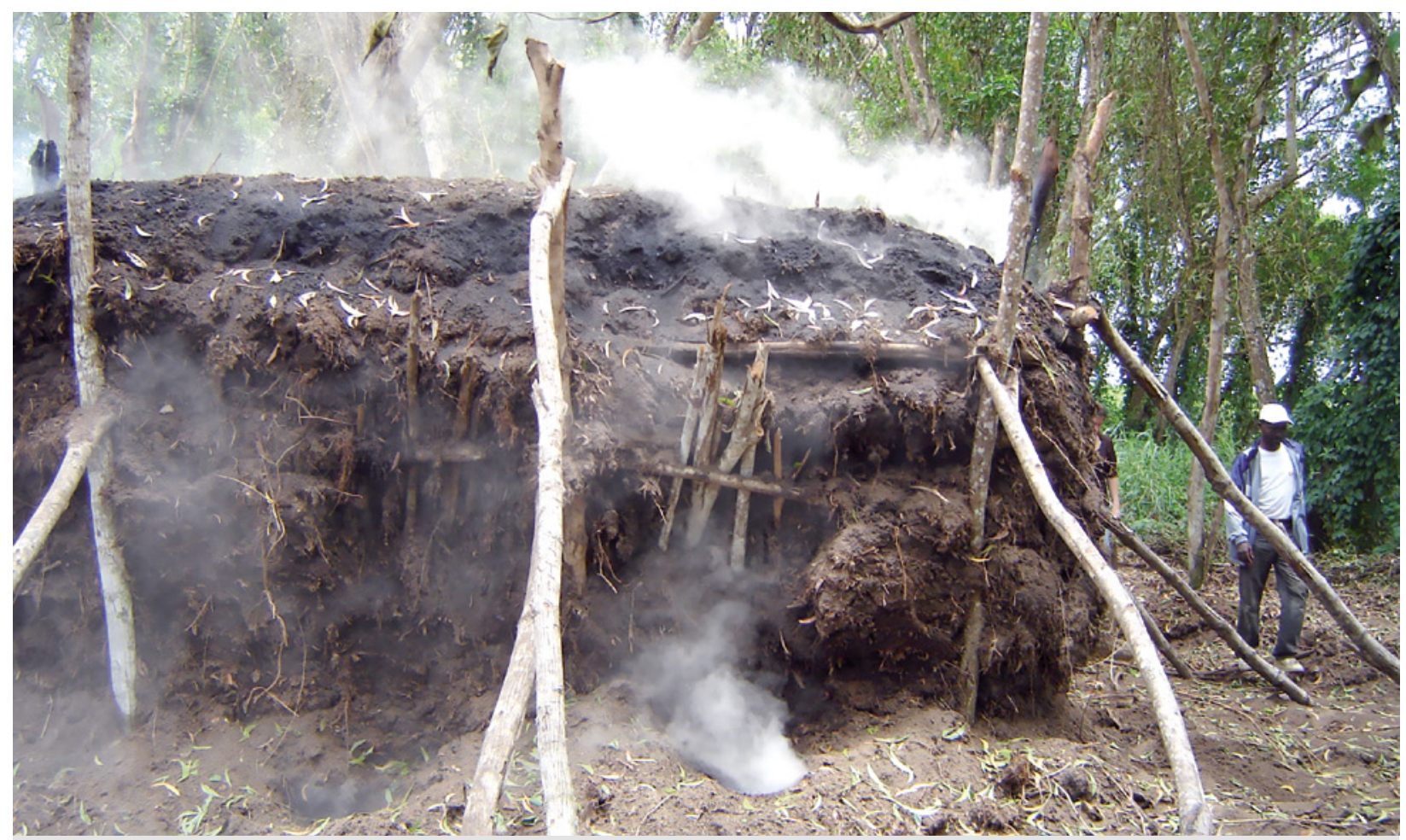

Photo 3.

À Mampu, en République démocratique du Congo, en 2009, un ingénieur du projet UE Makala forme les charbonniers à une technique de meule améliorée. Des techniques de fours métalliques fixes avaient été préalablement testées, mais n'avaient pas été adoptées du fait des difficultés de transport du bois. Photo R. Peltier. 
Quantité d'énergie domestique (par ex. en tonnes équivalent bois) disponible dans le bassin

d'approvisionnement (courbes vertes) et besoins d'une ville (courbes rouges)
Déficit (Productivité RN - Consommation ED)

Couvert par augmentation productivité RN

(gestion + RNA), économie et substitution d'ED,

Aménagements régionaux (1) \& politiques nationales (2)

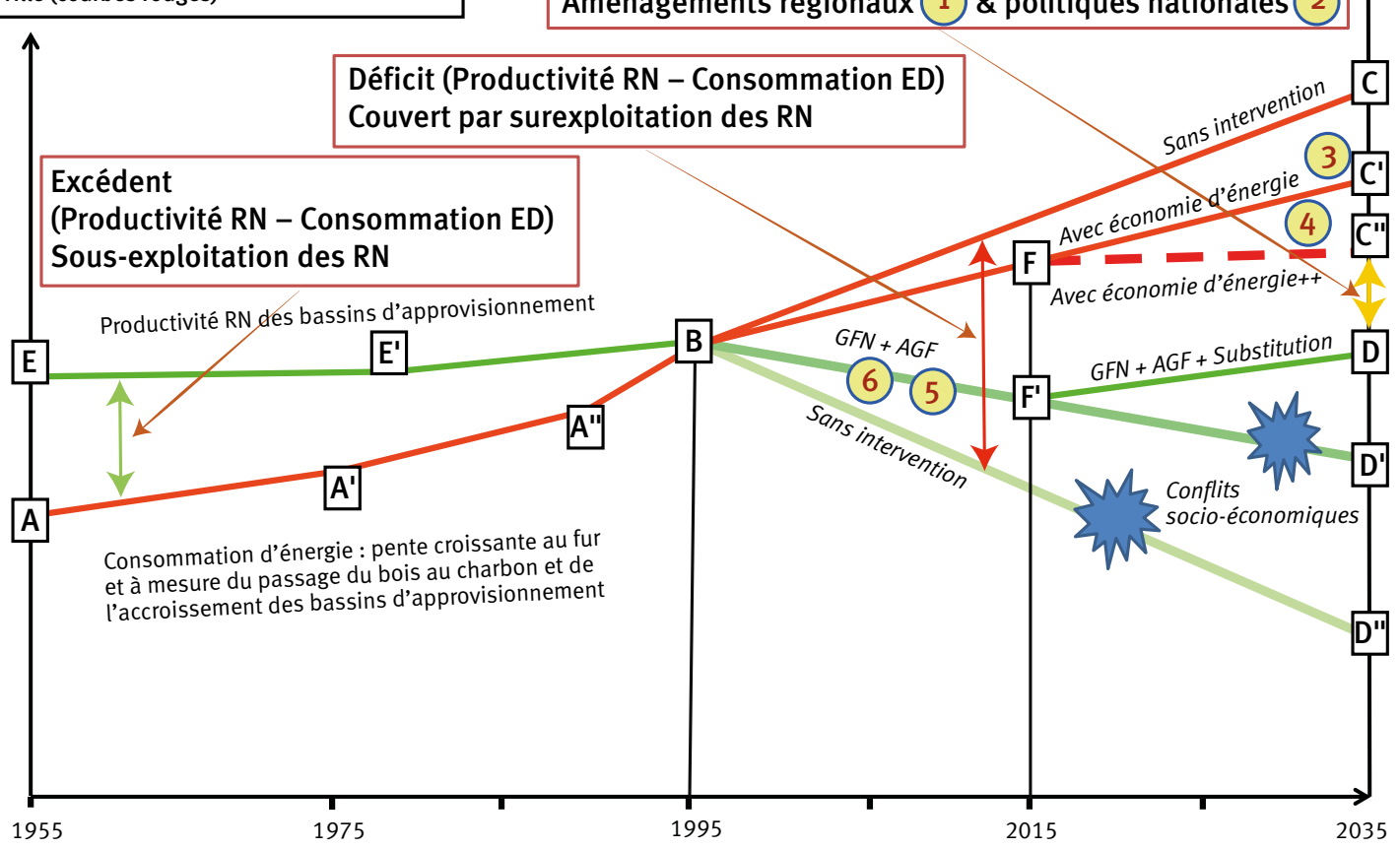

Figure 1.

Quelles évolutions pour la consommation d'énergie domestique (ED) et la gestion des ressources naturelles (RN) dans les bassins d'approvisionnement des grandes villes du Sud ? Notre vision dans un schéma prospectif. Lire les commentaires dans le texte pour la description des points d'inflexion (A à $F$ ). Les bulles numérotées en rouge et jaune correspondent aux articles venant conforter ou rejeter les hypothèses et le schéma prospectifs (1 : Charpin et al., $2019 ; 2$ : Sola et al., 2019 ; 3 : Temmerman et al., 2019 ; 4 : Schure et al., 2019 ; 5 : Péroches et al., 2019 ; 6 : Sibelet et al., 2019).

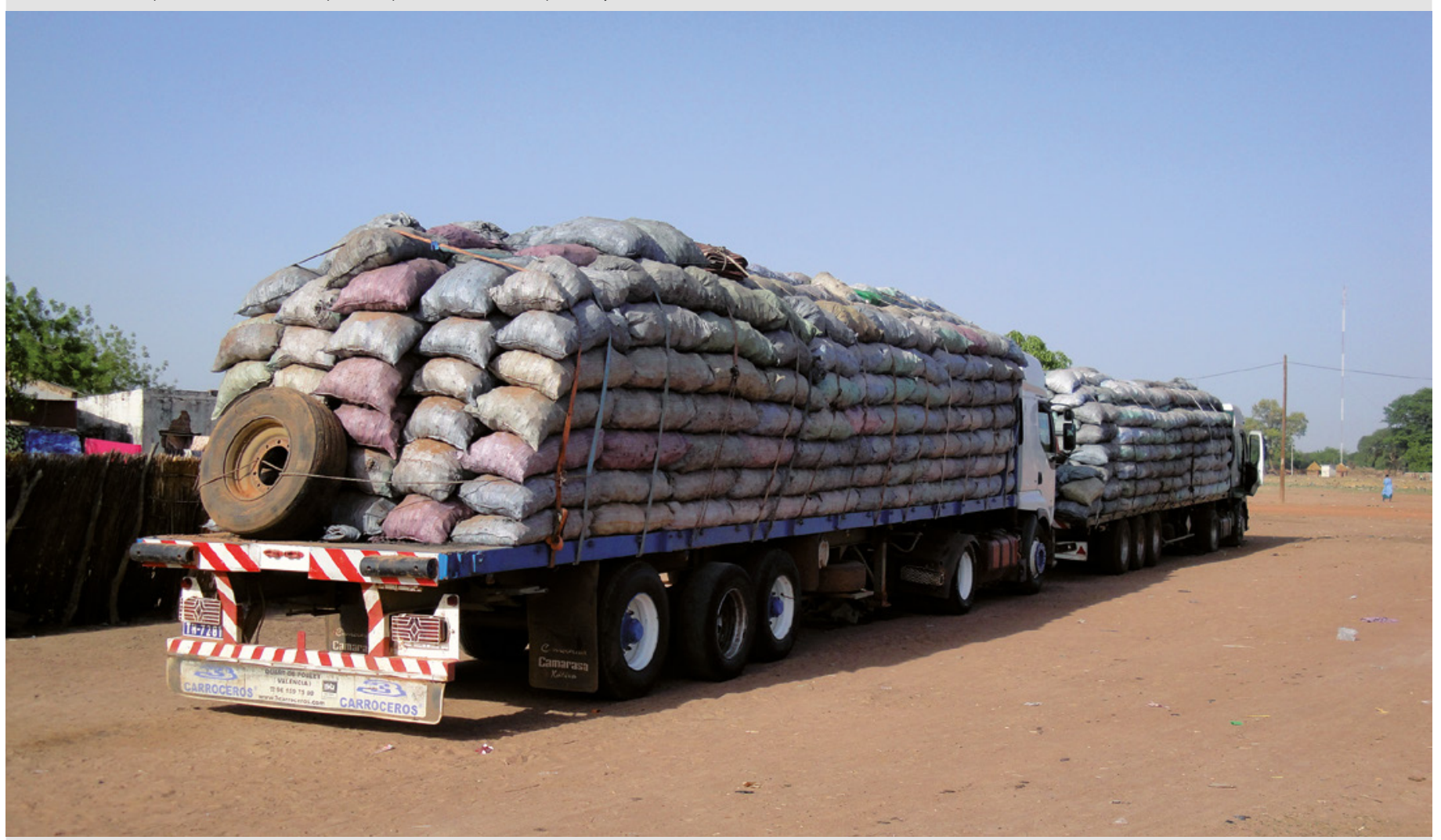

Photo 4.

Au Sénégal, d'énormes semi-remorques transportent le charbon de bois, depuis le sud-est du pays jusqu'à Dakar.

En 2012, le bassin d'approvisionnement de cette ville avait un rayon de $500 \mathrm{~km}$.

Photo R. Peltier. 


\section{De 1995 à nos jours: diverses tentatives pour freiner la dégradation des ressources naturelles menacées par la surexploitation du bois}

Avec l'augmentation de la population des villes, égale, voire supérieure à celle de la période précédente pour différentes raisons (exemple : effet réduit du planning familial, exode rural accéléré par le manque de terres et la péjoration climatique, arrivée de réfugiés liés aux conflits), les besoins en énergie domestique ont continué à augmenter (Montagne et al., 2016). En l'absence de toute action, une croissance linéaire pouvait être attendue (figure 1, segment $[B C]$ ). En faisant des économies d'énergie contraintes par le manque de ressources des citadins, tels la réduction du nombre de repas ou le passage à des plats nécessitant moins de cuisson, ou encouragées par les services gouvernementaux, les ONG et les projets diffusant des foyers améliorés, la pente de cette

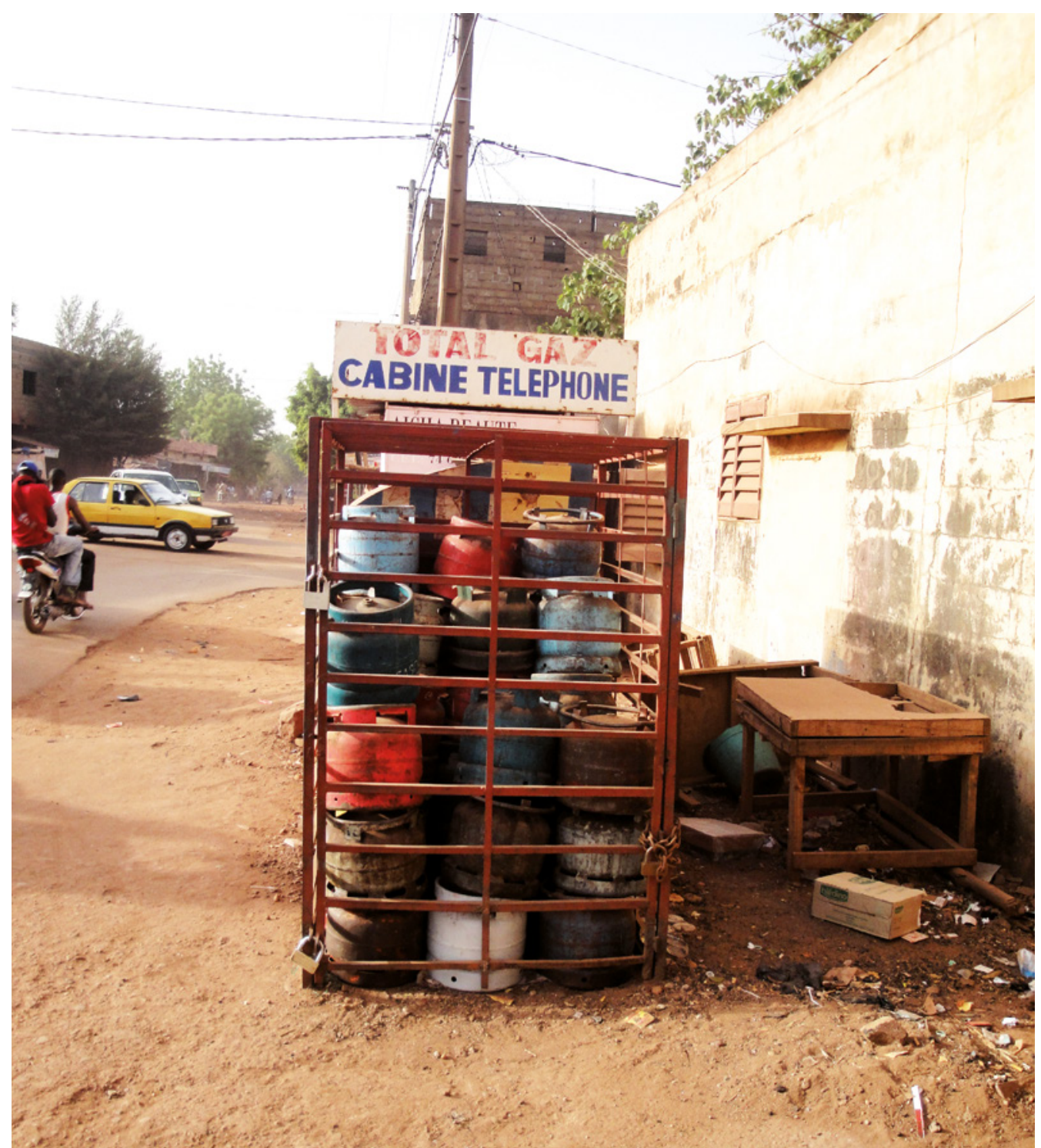

Photo 5.

Vente de bouteilles de gaz dans un quartier de Bamako, en 2015. Dans cette ville, grâce aux subventions de l'État malien, la substitution partielle du bois-énergie est déjà en route.

Photo R. Peltier. courbe a pu être légèrement atténuée (figure 1, segment [BF]). Mais les résultats ont été limités par une adoption réduite des foyers améliorés (Cirad-IDA-BEIE-CTFC, 2015a) et par une substitution d'énergie, en particulier avec le passage au gaz, limitée aux villes ayant adopté une politique vigoureuse de subvention (exemple : Bamako, Dakar) (photo 5).

Pour ce qui concerne la productivité de la ressource pendant cette période, la surexploitation des massifs au-delà de leur capacité de production a entraîné la baisse de leur productivité (Ichaou, 2000 ; Ichaou et al., 2015). Là où rien n'a été fait pour améliorer la gestion, la courbe a plongé (figure 1 , segment [BD"]) et la coupe de bois a non seulement touché les forêts, mais également les arbres des parcs arborés agroforestiers et parfois des vergers (Boubacar et al., 2017). Par contre, dans les pays qui ont développé des actions de gestion des forêts naturelles (GFN) (Mahamane et Montagne, 1997 ; Rives et al., 2013) et de restauration des systèmes agroforestiers (AGF) comme cela a été le cas au Niger (CiradIDA-BEIE-CTFC, 2015b), dans le cadre de politiques forestières intégrées (Projet Énergie II, 1993 ; Bertrand et Montagne, 2006), cette dégradation a été moins marquée (figure 1 , segment [BF']). Cependant, dans aucun pays, il n'a été possible d'augmenter la productivité de tout le bassin d'approvisionnement. Les raisons sont la faible surface de forêts aménagées et l'essoufflement de la politique de régénération naturelle assistée (RNA) des parcs agroforestiers, probablement dû au manque de subventions et à l'impérieuse nécessité, pour de nombreux paysans, de favoriser le présent (vente du bois) aux dépens du futur (restauration du parc).

De ce fait, dans la plupart des bassins d'approvisionnement, le déficit entre la capacité de production en bois-énergie des ressources naturelles et la quantité réellement récoltée (productivité $\mathrm{RN}$ - consommation ED) a été couvert par la surexploitation des RN (Peltier et al., 2009) et donc par le grignotage du capital bois sur pied (Neufeldt et al., 2015).

La flèche verticale rouge a la même vocation que pour la période précédente. La couleur rouge a été choisie pour exprimer que cette différence est fortement négative, ce qui implique une très forte dégradation des ressources naturelles. 


\section{Pour les vingt prochaines années, un avenir entre les mains de tous les acteurs}

Sur la base des observations des quatre dernières décennies et en prolongeant les courbes du schéma prospectif, nous pouvons émettre les hypothèses suivantes :

- Hypothèse 1. Dans le cas où les États et la communauté internationale laisseraient les ressources naturelles se dégrader sans intervention (figure 1, segment [BD']) et la consommation augmenter également sans intervention (figure 1, segment $[\mathrm{BC}]$ ), il est fort possible que l'énergie domestique devienne si chère dans les villes qu'à terme relativement court, la partie la plus fragile de la population se retrouve affamée et, par conséquent, acculée à la révolte ou à l'exil.

- Hypothèse 2. Des interventions timides, sur la lancée de celles qui sont en cours, en matière d'économie d'énergie (figure 1, segment [FC']) et de restauration de l'environnement (figure 1 , segment [F'D']), ne pourront que retarder ces troubles sociaux.

- Hypothèse 3. En conséquence, pour éviter des troubles sociaux urbains liés à la pénurie d'énergie domestique, tout en continuant à garantir des revenus aux ruraux par la vente de bois-énergie et la production de biens et services fournis par les espaces forestiers, agroforestiers et sylvo-pastoraux (Gado Alzouma, 2006 ; Schure, 2014), il est indispensable, d'une part, de combiner une politique d'économie d'énergie plus performante (figure 1 , segment [FC']) et, d'autre part, de lancer une politique très vigoureuse pour restaurer les éco systè mes, systèmes agroforestiers et sylvo-pastoraux dégradés. En outre, il faut gérer durablement les formations naturelles et substituer la partie de l'énergie domestique

Photo 6. Photo R. Peltier. qui dépasse les capacités de production des bassins d'approvisionnement en bois-énergie (figure 1, segment [F'D]). Ceci nécessitera également de faire évoluer les politiques nationales sur le foncier, les taxes et les subventions et d'établir des schémas d'aménagement régionaux. Il faudra enfin mobiliser tous les acteurs de la société civile et des organismes gouvernementaux des pays considérés. Dans le cadre de la décentralisation, en cours dans plusieurs pays, les communes auront un rôle important à jouer. En tant qu'utilisatrices de l'énergie domestique, mais également en tant que productrices de bois ou responsables des aspects sociaux dans les organisations de producteurs, et bien entendu en tant que mères de famille particulièrement attentives à l'avenir de leurs enfants, les femmes auront un rôle majeur à assumer (photo 6).

Sur notre schéma, la flèche verticale a la même signification que pour les deux périodes précédentes. La couleur orange a été choisie pour exprimer qu'il est à craindre que cette différence soit toujours négative, malgré les efforts entrepris. Ceux-ci porteront leurs fruits, mais cela risque d'être insuffisant pour permettre la restauration des ressources naturelles du bassin d'approvisionnement de la ville considérée. Il faudrait donc que des efforts supplémentaires soient assurés dans un avenir proche.

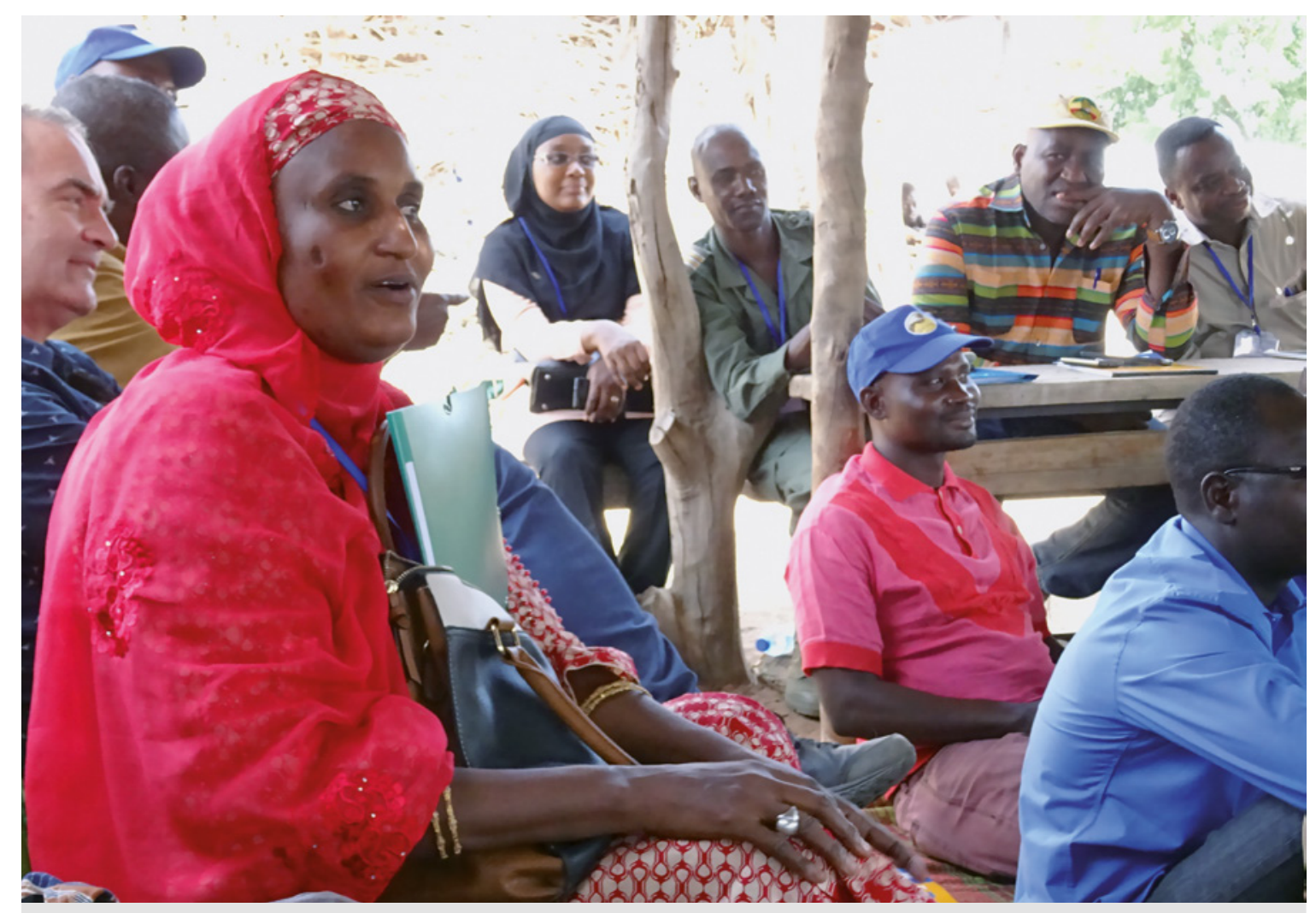

Au premier plan, Mme la vice-maire de la commune rurale de Say. Les femmes ont un rôle primordial à jouer, tant dans le domaine des économies d'énergie domestique que dans ceux de la gestion des ressources naturelles, de l'utilisation optimale des revenus du bois en milieu rural et de l'éducation des enfants. 


\section{En quoi les articles de ce numéro confirment ou infirment le modèle et les hypothèses}

Le numéro d'apparition de chacun des six articles a été reporté dans un cercle jaune sur la figure 1, de façon à montrer à quel niveau ils apportent des informations nouvelles.

Charpin et al. (2019) [1] caractérisent les filières bois-énergie autour d'Antananarivo (Madagascar). Le bois est utilisé par $90 \%$ des ménages, dont $70 \%$ utilisent des foyers améliorés. Le taux de réduction du couvert forestier est en forte augmentation, malgré la présence de 70000 ha de plantations d'eucalyptus et 120000 ha de systèmes agroforestiers. Cette prépondérance du bois-énergie s'explique par le manque d'attractivité, de compétitivité et d'accessibilité des alternatives énergétiques. La pauvreté d'une part importante de la population participe au maintien d'un prix de vente bas aux consommateurs, qui ne permet pas une gestion durable des ressources forestières. Mais les initiatives de régulation des flux de bois-énergie sont rendues difficiles, du fait des risques de mouvements sociaux qu'elles peuvent engendrer. Le premier Schéma régional d'approvisionnement en bois-énergie (SRABE) a été élaboré sous l'égide de la région d'Analamanga. Celui-ci permet l'identification, la priorisation et la planification des actions ayant pour objectif de sécuriser l'approvisionnement, de favoriser les plantations, d'accompagner la formalisation des acteurs économiques, et de favoriser une utilisation plus efficiente des combustibles domestiques. Au regard de la réussite de ces actions concertées, des SRABE doivent être élaborés très prochainement dans trois autres régions qui font partie du bassin d'approvisionnement de la capitale.

Dans le deuxième article, Sola et al. (2019) [2] comparent les politiques et les mécanismes institutionnels dans le secteur du bois-énergie de quatre pays africains avec la réalité du fonctionnement des filières. Ils constatent que, malgré son importance, la place du bois-énergie a été faiblement prise en compte au plan politique et institutionnel, car il était supposé que le développement économique allait permettre la substitution de cette énergie vers d'autres, plus modernes. Or, la pauvreté et le manque d'énergies abordables n'ont pas permis cette transition. L'importance du bois-énergie est, malgré tout, de mieux en mieux reconnue, en général associée à la dégradation forestière et à des bénéfices socio-économiques pour les populations les plus pauvres. Mais les lois sont en général contournées. Le secteur du bois-énergie reste dans le secteur informel, est victime de la corruption et ne bénéficie que d'investissements trop faibles ou inadéquats. Les auteurs militent pour que les gouvernements prennent ce problème à bras-le-corps en mettant en place un corpus de lois et de règles mieux adapté aux réalités des pays.

Temmerman et al. (2019) [3] présentent quant à eux une étude portant sur une technique d'amélioration du rendement d'un four de carbonisation du bois à Madagascar. Cet article montre clairement qu'il serait techniquement possible de consommer moins de bois, et donc de dégrader moins les ressources naturelles, tout en fournissant la même quantité d'énergie domestique à la population et en limitant l'émission de gaz à effet de serre, en particulier du méthane. C'est donc une technique qui mérite d'être testée à plus grande échelle, tout en étudiant les facteurs socio-économiques qui pourraient en limiter la diffusion. Son coût allégé, $3000 €$ pour un four Green Mad Retort version 3, peut certainement être facilement amorti par le gain de productivité sur des durées de vie longues, sachant qu'un tel four fonctionne depuis déjà 6 ans à Madagascar, et avec des durées courtes du cycle de carbonisation d'environ une semaine.

Schure et al. (2019) [4] explorent les facteurs autres que techniques qui limitent l'efficacité de la production de charbon de bois en Afrique subsaharienne. Cela les amène à étudier le contexte institutionnel et les capacités des acteurs de la filière. En prenant l'exemple de deux régions de la République démocratique du Congo, ils montrent que des gains importants peuvent être obtenus en améliorant les techniques de carbonisation. Cependant, le succès dépend de toute une chaîne institutionnelle qui comprend, entre autres, la légalisation de toute la filière, une simplification des taxes, un appui à l'organisation des producteurs, leur accès au crédit et à la formation, et une rémunération équitable des différents acteurs.

Péroches et al. (2019) [5] décrivent comment, afin d'améliorer la gestion des espaces périurbains et d'assurer l'alimentation durable en bois-énergie de Kinshasa, le projet UE Makala a co-construit, avec douze communautés du Plateau Batéké et du Kongo central, des plans simples de gestion (PSG) des terroirs villageois. Les itinéraires techniques de plantations agroforestières testés avec les agriculteurs producteurs de bois-énergie de ces communautés sont également présentés. L'appropriation des PSG et des itinéraires techniques a été évaluée un an et demi après la fin du projet. Les auteurs constatent que ces innovations ont été mieux adoptées dans les zones où le bois est devenu rare, lorsque les bénéficiaires des futurs produits ont été clairement identifiés et quand les droits fonciers ont été précisément établis.

Enfin, dans le dernier article, Sibelet et al. (2019) [6] précisent les effets du développement de systèmes agroforestiers au Guatemala sur la satisfaction des besoins en bois-énergie de la population rurale et sur l'amélioration de ses moyens d'existence. Le système agroforestier enrichi, de type « culture en couloirs », permet de couvrir $65 \%$ des besoins des ménages, contre $7 \%$ pour le système traditionnel. Ils émettent l'hypothèse que ceci réduit considérablement les prélèvements en forêt naturelle et sa dégradation. Les agriculteurs qui ont adopté le système introduit par un projet FAO, basé sur Gliricidia sepium, l'ont fait évoluer en fonction de leurs savoirs et de leurs besoins, en y introduisant des fruitiers, des arbres producteurs de bois d'œuvre, etc. Cela a fortement amélioré les moyens et les conditions d'existence des agriculteurs, mais la sécurité foncière reste un préalable indispensable pour le développement de ces systèmes.

Brièvement, nous allons voir en quoi ces articles contribuent à conforter ou à infirmer notre schéma prospectif et nos hypothèses. 
L'article de Charpin et al. (2019) conforte parfaitement notre hypothèse 3 concernant la nécessité d'établir des schémas régionaux d'aménagement et de les lier à une politique vigoureuse (figure 1 , segments [FC'] et [F'D]). Toutefois, il apparaît que, dans le cas d'une ville comme Antananarivo, l'extrême pauvreté de la population, en grande partie liée à la sous-industrialisation du pays, complique considérablement la recherche de solutions durables. En effet, la population ne peut ni payer le bois à son juste prix, ni le remplacer par des énergies alternatives. En outre, l'article de Sola et al. (2019) montre que la mauvaise gouvernance de nombreux pays africains limite fortement l'impact des initiatives concernant la législation et la taxation. Ce n'est donc pas le fait de maintenir des filières bois-énergie qui est responsable du maintien des bûcherons et d'autres agents de la filière dans la pauvreté. C'est au contraire la pauvreté des urbains et la mauvaise gouvernance qui obligent à souspayer le bois et le charbon, et donc à empêcher les investissements nécessaires pour intensifier et rendre plus durable l'amont de la filière, comme cela existe dans des pays plus développés. Cela montre que les solutions à la crise de l'énergie domestique devront également être recherchées en dehors des seules filières du bois-énergie.

L'article de Temmerman et al. (2019) révèle clairement qu'il existe déjà des techniques permettant d'augmenter fortement le rendement de carbonisation et donc de limiter les prélèvements de bois, pour fournir une même quantité d'énergie domestique (figure 1, segment [BF]). Cependant, si ce type de four fixe convient bien pour la carbonisation de bois produit par des plantations d'espèces à croissance rapide, sur des terrains où le débardage par traction animale ou mécanique est possible, il est beaucoup plus difficile, voire impossible, de le diffuser dans des forêts naturelles ou des jachères dispersées au sein de territoires au sol accidenté et auprès de paysans essarteurs très pauvres. Ce type d'innovation peut clairement contribuer à réduire les besoins en biomasse. Mais dans un avenir proche, il ne concernera qu'une petite part des producteurs artisanaux de charbon, dans des pays comme le Niger ou la République démocratique du Congo. Ce type d'innovation contribuera à faire baisser la pente du segment [FC'] (figure 1) mais cela ne suffira pas, d'autant plus que l'article de Schure et al. (2019) nous rappelle que c'est tout l'environnement de la filière charbon de bois qu'il faudra faire évoluer.

Péroches et al. (2019) et Sibelet et al. (2019) nous amènent à constater que des innovations techniques et d'organisation des communautés peuvent limiter la dégradation des ressources naturelles, voire les restaurer et en améliorer la productivité. Cependant, il apparaît que les populations n'acceptent d'intensifier leurs pratiques que lorsqu'elles y sont contraintes par la pénurie de ressources, avec l'appui d'organisations extérieures qui nécessitent un investissement, et avec l'appui des autorités gouvernementales. La nécessité de sécuriser le foncier est également mise en avant. Cela conforte bien le fait qu'il a été possible, au cours de la dernière décennie, de ralentir les processus de dégradation des ressources naturelles (figure 1 , segment [BF']). Cela valide également la partie de notre hypothèse 3 stipulant qu'il est nécessaire de lancer une politique très vigoureuse pour restaurer les écosystèmes, systèmes agroforestiers et sylvo-pastoraux dégradés.

Nous pouvons regretter que nous n'ayons pas été en mesure de publier des articles concernant la substitution d'énergie, la dynamique agroforestière et les schémas directeurs d'approvisionnement en bois-énergie au Sahel. Nous espérons vivement que des articles sur ces sujets et bien d'autres concernant le bois-énergie parviendront à la revue Bois et Forêts des Tropiques dans les mois à venir.

\section{Références bibliographiques}

Bachard L., 2005. Décentralisation et développement local durable : Étude de la gestion des ressources forestières et du bois énergie au Niger. Mémoire Université Senghor, Alexandrie, Égypte, 70 p.

Bertrand A., Montagne P., 2006. Les difficiles mutations des politiques forestières : d'une gestion autoritaire et exclusive vers une politique publique intégrée. In : Bertrand A., Montagne P., Karsenty A. (éds). L'état et la gestion locale durable des forêts en Afrique francophone et à Madagascar. Paris, France, L'Harmattan, 37-53.

Boubacar A., Gafsi M., Sibelet N., Adam T., Peltier R., Oumarou I., et al., 2017. Ressources arborées et viabilité des exploitations agricoles familiales dans le bassin en bois-énergie de Niamey (Niger). Colloque national « Gestion des ressources forestières des territoires périurbains et changement climatique », 31 octobre - 2 novembre 2017, Niamey, Niger, 18 p. http://agritrop.cirad.fr/587691/

Charpin M., Legeay D., Rabemanantsoa N., Richter N., 2019. Caractérisation des filières bois-énergie et élaboration du schéma d'approvisionnement en bois-énergie de la région d'Analamanga, Madagascar. Bois et Forêts des Tropiques, $340: 13-25$.

Cirad-IDA-BEIE-CTFC, 2015a. Schéma directeur d'approvisionnement en combustibles domestiques de Maradi et Zinder. Washington, USA, Banque mondiale, Technical document.

Cirad-IDA-BEIE-CTFC, 2015b. Programme national des énergies domestiques du Niger. Washington, USA, Banque mondiale, Technical document.

Gado Alzouma R., 2006. Contribution des aménagements forestiers au développement des communautés locales : Cas des marchés ruraux de bois-énergie du massif de Tientiergou, dans le Département de Say. Mémoire de maîtrise en sociologie, Université Abdou Moumouni, Niamey, Niger, $58 \mathrm{p}$.

Gazull L., 2009. Le bassin d'approvisionnement en bois-énergie de Bamako. Une approche par un modèle d'interaction spatiale. Thèse de doctorat, Université Paris-Diderot - Paris VII, France, 423 p. https://tel. archives-ouvertes.fr/file/index/docid/670473/filename/ These LG Finale 100211.pdf 
Gazull, L., Gautier D., 2014. Woodfuel in a global change context. WIREs Energy and Environment, 4 (2): 156-170. https://doi.org/10.1002/wene.115

Ichaou A., 2000. Dynamique et productivité des structures forestières contractées des plateaux de l'Ouest nigérien. Thèse de doctorat, Université Paul Sabatier Toulouse III, France, 231 p. http://agritrop.cirad.fr/575097/

Ichaou A., 2004. Aménagement participatif et gestion décentralisée des forêts naturelles pour la production de bois-énergie : capitalisation de l'expérience nigérienne. PREDAS CILSS, $36 \mathrm{p}$.

Ichaou A., Malam Issa A., Montagne P., Peltier R., 2015. Bois-énergie des villes sahéliennes, évolution de l'approvisionnement en combustibles domestiques de 1993 à 2013. Cas de Maradi et de Zinder, au Niger. In: WFC2015, FAO World Forestry Congress: Forests and People, Investing in a Sustainable Future, Durban, South Africa, 7-11 September 2015, poster.

Ichaou A., Peltier R., 2017. Gestion multi-usages, socialement et écologiquement durable des écosystèmes forestiers nigériens. In : Colloque international « Gestion des ressources forestières des territoires périurbains et changement climatique », Niamey, Niger, 31 octobre - 2 novembre 2017, 15 p. https://agritrop.cirad.fr/590826/

Madon G., Matly M., Bertrand A., Laurent D., 1985. La filière bois à Niamey. Projet forestier IDA/FAC/CCCE, SEMA-énergie-Cirad-CTFT.

Mahamane L., Montagne P., 1997. Les grands axes stratégiques du Projet Énergie II - Volet offre pour une gestion rationnelle des écosystèmes forestiers péri-urbains au Niger. In : D’Herbès J. M., Ambouta J. M. K., Peltier R. Fonctionnement et gestion des écosystèmes forestiers contractés sahéliens. Montrouge, France, John Libbey Eurotext, 155-167. https://ur-forets-societes.cirad.fr/ content/download/4431/35723/

Marien J.-N., Peltier R., Dubiez E., Trefon T., 2018. Wood: an ever-present domestic energy priority for people in emerging Africa. International Conference on Sustainable Energy for Africa, Belgium, 23-25 October 2017, 32 p. http://www. kaowarsom.be/documents/Energy4Africa/SustainableEnergy4Africa Peltier.pdf

https://www.kaowarsom.be/en/SustainableEnergy4Africa presentations $\% 26$ videos

Montagne P., Oumarou I., Bertrand A., Rives F., Ichaou A., Peltier R., 2016. Bois-énergie domestique, démographie et urbanisation : situation après vingt-cinq années de gestion forestière des néo-communs au Sud-Niger. In : Communs et Développement. Paris, France, AFD, 14 p. http://agritrop. cirad.fr/582613/

Neufeldt H., Dobie P., liyama M., Njenga M., Mohan S., Neely C., 2015. Developing sustainable tree-based bioenergy systems in sub-Saharan Africa. Kenya, World Agroforestry Centre (ICRAF), Policy Brief, 28, 8 p. https://www.globallandscapesforum.org/publication/developing-sustainabletree-based-bioenergy-systems-in-sub-saharan-africa/
Peltier R., Lawali E. M., Montagne P., 1994. Aménagement villageois des brousses tachetées au Niger. 1 ère partie - Le milieu : potentiel et contraintes. Bois et Forêts des Tropiques, 242 : 59-76. http://revues.cirad.fr/index.php/BFT/ article/view/19804

Peltier R., Ichaou A., Gado Alzouma R., Dessard H., 2009. Bilan après quinze ans de gestion communautaire d'une forêt villageoise de l'Ouest nigérien. Évolution de la ressource arborée et de la perception des populations. Sécheresse, 20 (4) : 20-31. http://www.secheresse.info/IMG/pdf/ vol20 n1e Peltier.pdf

Péroches A., Dubiez E., Peltier R., Proces P., Diowo S., Yamba-Yamba T., et al., 2019. Les plans simples de gestion destinés à la production de bois-énergie en périphérie de Kinshasa : la participation et la restauration à l'épreuve du foncier. Bois et Forêts des Tropiques, 340 : 71-90.

Projet Énergie II - Énergie domestique, 1993. Schéma directeur d'approvisionnement en Bois-énergie de Maradi et de Zinder. Seed/Cirad-Forêt, 112 p.

Rives F., Peltier R., Montagne P., 2013. Fifteen years of forest community management in Niger: From a technician's dream to social reality. Small-scale Forestry, 12 (1): 93-105. https://doi.org/10.1007/s11842-012-9203-5

Sibelet N., Posada K. E., Gutiérrez-Montes I. A., 2019. Agroforestry systems providing firewood toward livelihood improvement in Guatemala. Bois et Forêts des Tropiques, 340: 91-102.

Schure J., 2014. Woodfuel for urban markets in the Congo Basin: a livelihood perspective. PhD thesis, Wageningen University, Netherlands, 186 p. https://library.wur.nl/WebQuery/wda/2048558

Schure J., Pinta F., Cerutti P. O., Kasereka-Muvatsi L., 2019. Efficiency of charcoal production in Sub-Saharan Africa: Solutions beyond the kiln. Bois et Forêts des Tropiques, 340: 57-70.

Sola P., Schure J., Eba'a Atyi R., Gumbo D., Okeyo I., 2019. Woodfuel policies and realities in Sub-Saharan Africa. Bois et Forêts des Tropiques, 340: 27-41.

Temmerman M., Andrianirina R., Richter F., 2019. Exemple technique d'amélioration du rendement d'un four de carbonisation du bois à Madagascar. Bois et Forêts des Tropiques, $340: 43-55$.

Bois et Forêts des Tropiques - Revue scientifique du Cirad
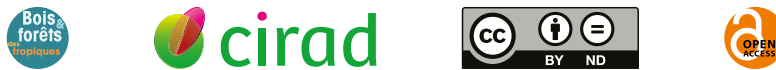

Cirad - Campus international de Baillarguet, 34398 Montpellier Cedex 5, France - Contact : bft@cirad.fr - ISSN : L-0006-579X 